\title{
Chimeric antibody targeting SRPK-1 in the treatment of non-small cell lung cancer by inhibiting growth, migration and invasion
}

\author{
FAN WU, JIE LI, XIN DU, WEISAN ZHANG, PING LEI and QIANG ZHANG \\ Department of Geriatrics, Tianjin Medical University General Hospital, Tianjin 300052, P.R. China
}

Received March 9, 2016; Accepted February 2, 2017

DOI: $10.3892 / \mathrm{mmr} .2017 .6833$

\begin{abstract}
Non-small cell lung cancer (NSCLC) is one of the most common types of cancer in humans, and is characterized by rapid growth, migration, invasion and reoccurrence. Evidence has indicated that the protein and mRNA levels of serine-arginine protein kinase-1 (SRPK-1) are upregulated in NSCLC tissues. However, the functions of SRPK1 and targeted therapy for SRPK1 in the progression and treatment of NSCLC remain to be fully elucidated. In the present study, the mRNA and protein expression levels of SRPK-1 in NSCLC cells and tissues were analyzed using reverse transcription-quantitative polymerase chain reaction analysis and SDS-PAGE, and the role of SRPK1 in the progression of NSCLC was investigated. In addition, a chimeric antibody target for SRPK-1 (ChanSRPK-1) was constructed, and the therapeutic effects of ChanSRPK-1 were investigated in H358-bearing mice. The curative effects of ChanSRPK-1 on the inhibition of growth, migration and invasion of NSCLC were also examined in vitro and in vivo. The results revealed that the mRNA and protein levels of SRPK-1 were upregulated in NSCLC cells and tumor tissues. Higher expression of SRPK1 promoted NSCLC cell growth, migration and invasion, whereas lower expression of SRPK-1 suppressed growth, migration and invasion of the NSCLC cells. Animal experiments demonstrated that ChanSRPK-1 inhibited the $\beta$-catenin/T-cell factor complex. ChanSRPK-1 treatment also downregulated the phosphorylation levels of glycogen synthase kinase $3-\beta$ and prolonged the survival of tumor-bearing mice. Taken together, SRPK-1 may offer potential as a therapeutic target oncogenic molecular in NSCLC, and ChanSRPK-1 may be a therapeutic agent with functions as a target and for oncolytic therapy in the treatment of NSCLC.
\end{abstract}

Correspondence to: Professor Qiang Zhang, Department of Geriatrics, Tianjin Medical University General Hospital, 154 Anshan Road, Tianjin 300052, P.R. China

E-mail: qiangzhangprof@yeah.net

Key words: non-small cell lung cancer, serine-arginine protein kinase-1, chimeric antibody target for serine-arginine protein kinase-1, target therapy, oncolytic therapy

\section{Introduction}

Non-small cell lung cancer (NSCLC) is one of the most common types of cancer, owing to poor air quality and environmental pollution in the world (1). A previous study reported that tumor growth, migration and invasion in NSCLC are the most important features in tumor metastasis, development and reoccurrence (2). The occurrence of NSCLC has increased due to industrial pollution and the degradation of the ecological environment in the last century $(3,4)$. NSCLC comprises adenocarcinoma, large cell carcinoma and squamous cell carcinoma, and is the most frequent type of lung cancer, which accounts for $\sim 80 \%$ of all lung cancer cases (5-7). Despite increasing therapeutic improvements for NSCLC, the survival rates of patients with NSCLC are poor, with overall 5 -year survival rates of $<15 \%$, indicating it a critical clinical problem $(6,8,9)$. In addition, the migration and invasion of NSCLC are the primary reasons for the poor survival rates in treatment and recurrence for patients with $\operatorname{NSCLC~}(10,11)$. Therefore, inhibiting the migration and invasion of NSCLC may offer potential as an efficient therapeutic schedule for patients with cancer $(12,13)$.

Li et al reported that an increase in a pre-mRNA splicing regulator for serine-arginine protein kinase-1 (SRPK-1) increased cancer development and metastasis (14). Clinical investigations of the expression of SRPK-1 in NSCLC have also been performed, the results of which indicated that SRPK-1 exerted an oncogenic function in NSCLC and suggested that SRPK-1 may serve as a therapeutic target for patients with NSCLC (15). The increased expression of SRPK-1 is correlated with disease grade in several types of tumor, including retinoblastoma, breast cancer, pancreatic cancer and colon cancer (15-18). In the present study, a chimeric antibody target for SRPK-1 (ChanSRPK-1) was constructed and used to examine whether ChanSRPK-1 showed beneficial efficacy on the increased expression of SRPK1 in an NSCLC mouse model. The present study also evaluated the possible long-term survival of NSCLC-bearing mice, and investigated the therapeutic effects on expression of the $\beta$-catenin/T-cell factor (TCF) complex and the phosphorylation levels of glycogen synthase kinase 3- $\beta$ (GSK3- $\beta$ ) in cancer-associated processes.

The development of effective drugs to inhibit tumor cell growth, migration and invasion in patients with NSCLC has already focused on public and personalized medication. 
A previous study indicated that SRPK-1 was specifically expressed in epithelial cells in normal breast and colon cells (18). Of note, ChanSRPK-1-mediated neutralization has been demonstrated as a novel and beneficial antibody in the progress and metastasis of NSCLC (19).

Although suppressing the expression of SRPK-1 in tumor cells has demonstrated beneficial outcomes, the use of anti-SRPK-1 antibody for NSCLC therapy has not been investigated. The present study aimed to investigate the expression of SRPK-1, and the inhibitory effects of ChanSRPK-1 on migration and invasion in an NSCLC mouse model. ChanSRPK-1 inhibited the mRNA expression levels of TCF, matrix metalloproteinase-9 (MMP), collagen type I (CT1) and fibronectin (FBC) in NSCLC-derived vascular endothelial cells (NSCLCDVECs). The present study also investigated the therapeutic outcomes and molecular mechanism via long-term treatment with ChanSRPK-1, which indicated that the migration and invasion of NSCLC were inhibited following treatment with ChanSRPK-1.

\section{Materials and methods}

Cell culture. The MRC-5 cells were purchased from American Type Culture Collection (Manassas, VA, USA). The NSCLCDVECs were a provided by the University of Toronto (Toronto, Canada). The MRC-5 cells and NSCLCDVECs were cultured in EMEM supplemented with $10 \%$ fetal bovine serum (FBS; Gibco; Thermo Fisher Scientific, Inc., Waltham, $\mathrm{MA}, \mathrm{USA}$ ) at $37^{\circ} \mathrm{C}, 5 \% \mathrm{CO}_{2}$ and reasonable humidity.

MTT assay. The NSCLCDVECs $\left(5 \times 10^{3}\right)$ were grown in 12 -well plates to $\sim 95 \%$ monolayer cells. Subsequently, either ChanSRPK-1 (400 mg/ml) or PBS was added to the plates for $24,48,72$ and $96 \mathrm{~h}$ at $37^{\circ} \mathrm{C}$, respectively. MTT, at concentration of $5 \mathrm{mg} / \mathrm{ml}$ (50 $\mu \mathrm{l}$; Amresco, LLC, Solon, OH, USA), was added to the cells and incubated for $4 \mathrm{~h}$. DMSO was added for incubation for $30 \mathrm{~min}$ to dissolve the precipitate following removal of the supernatant. The results were determined using a spectrophotometer (Bio-Rad Laboratories, Inc., Hercules, CA, USA) at $570 \mathrm{~nm}$.

Reverse transcription-quantitative polymerase chain reaction $(R T-q P C R)$ analysis. Total mRNA was isolated from the MRC-5 cells and NSCLCDVECs pre- and post-treatment with ChanSRPK-1 using an RNAeasy Mini kit (Qiagen Sciences, Inc., Gaithersburg, MD). The total mRNA (1 $\mu \mathrm{g})$ was reverse transcribed into cDNA using a reverse transcription kit (Qiagen Sciences, Inc.). The cDNA (10 ng) was used for qPCR (Bio-Rad Laboratories, Inc. Hercules, CA, USA) with the SYBR Green Master Mix system (50 ng of genomic DNA, $200 \mu \mathrm{M}$ dNTP, 2.5 units of Taq DNA polymerase, and $200 \mu \mathrm{M}$ primers) according to manufacturers' protocols, followed by preliminary denaturation at $94^{\circ} \mathrm{C}$ for $2 \mathrm{~min}$, 35 cycles at $94^{\circ} \mathrm{C}$ for $30 \mathrm{sec}$, annealing temperature reduced to $64^{\circ} \mathrm{C}$ for $30 \mathrm{sec}$ and $72^{\circ} \mathrm{C}$ for $10 \mathrm{~min}$. All forward and reverse primers (Table I) were synthesized by Invitrogen (Thermo Fisher Scientific, Inc.). Relative mRNA expression changes were calculated using the $2^{-\Delta \Delta \mathrm{Cq}}$ method (20). The results are expressed as the fold change, compared with the control.
Cell viability assay. The MRC-5 cells and NSCLCDVECs were grown in a 24 -well plate at a concentration of $2.5 \times 10^{5} / \mathrm{ml}$ (total column of 1,000 $\mu \mathrm{l}$ ). The MRC-5 cells and NSCLCDVECs were treated with ChanSRPK-1 $(400 \mathrm{mg} / \mathrm{ml})$ or PBS (control) for $48 \mathrm{~h}$ at $37^{\circ} \mathrm{C}$. The viabilities of the MRC-5 cells and NSCLCDVECs were examined using a Cell Counting kit (Dojindo Molecular Technologies, Inc., Kumamoto, Japan) in eight view windows.

Cell invasion and migration assays. The MRC-5 cells and NSCLCDVECs were treated with ChanSRPK-1 or PBS (control). For the invasion assay, the cells were suspended as a density of $1 \times 10^{5}$ in $500 \mu \mathrm{l}$ serum-free EMEM. Following treatment of the MRC- 5 cells and NSCLCDVECs with ChanSRPK-1 or PBS, the cells were added to the upper chamber of BD BioCoat Matrigel Invasion chambers (BD Biosciences, Franklin Lakes, NJ, USA) according to the manufacturer's protocol. For the migration assay, the cells were inoculated with ChanSRPK-1 or PBS for $48 \mathrm{~h}$ using a control insert (BD Biosciences) rather than a Matrigel invasion chamber. The tumor cells invasion and migration were counted in at least three randomly selected stained MRC-5 cells or NSCLCDVECs fields from every membrane under a light microscope (N-STORM, Nikon Corporation, Tokyo, Japan).

ELISA. The levels of SRPK-1 were determined using a commercial ELISA kit (CSB-EL022682MO, Cusabio Biotech Co., Ltd, Wuhan, China) for SRPK-1, and the procedure was performed according to the manufacturer's protocol. Briefly, ChanSRPK-1 $(20-420 \mu \mathrm{g} / \mathrm{ml}, 50 \mu \mathrm{l})$ was added to ELISA plates wells for $30 \mathrm{~min}$ at $37^{\circ} \mathrm{C}$. These were then washed three times with PBS and followed by blocking for $2 \mathrm{~h}$ with $300 \mu 15 \%$ non-fat dried milk in PBS. Then, $100 \mu 1$ horseradish peroxidase (HRP)-conjugated SRPK-1 $(0.2 \mu \mathrm{g} / \mathrm{ml})$ was added to the wells and incubated for $1 \mathrm{~h}$ at $37^{\circ} \mathrm{C}$. The plates were washed three times with PBS and absorbance was measured at $450 \mathrm{~nm}$ in an ELISA reader.

Western blot analysis. The NSCLCDVECs were treated with ChanSRPK-1 (400 ng/ml) or PBS for $48 \mathrm{~h}$. The NSCLCDVECs were then harvested by scraping and were lysed in RIPA buffer, followed by homogenization at $4^{\circ} \mathrm{C}$ for $10 \mathrm{~min}$. A total of $20 \mu \mathrm{g}$ protein extracts was electrophoresed on $12.5 \%$ polyacrylamide gradient gels and then transferred to nitrocellulose membranes. The membranes were incubated in blocking buffer (5\% milk) prior to incubation with primary antibodies at $4^{\circ} \mathrm{C}$ overnight. The ChanSRPK-1-treated and PBS-treated NSCLCDVECs were inoculated with the correlating mouse anti-human primary antibody: SRPK-1 (ab50927; 1:500), Cytochalasin-D (ab143484; 1:500), G-actin (ab123034; 1:500) and $\beta$-actin (ab5694; 1:500; all from Abcam, China) for $12 \mathrm{~h}$ at $4^{\circ} \mathrm{C}$. All samples were washed with Tris-buffered saline and incubated with HRP-conjugated bovine anti-mouse IgG antibodies (sc-2371; 1:10,000; Santa Cruz Biotechnology, Inc., Dallas, TX, USA) for $2 \mathrm{~h}$ at $37^{\circ} \mathrm{C}$. The results were visualized by using a chemiluminescence detection system (BD Biosciences).

Animal experiments. A total of 100 specific pathogen-free male BABL/C (six-week old, body weight: 32-36 g) mice were 
Table I. Primer sequences used for reverse transcription-quantitative polymerase chain reaction.

\begin{tabular}{lll}
\hline Target gene & \multicolumn{1}{c}{ Forward primer } & \multicolumn{1}{c}{ Reverse primer } \\
\hline SPRK-1 & 5'-GGACCGTCTTCGGACATCA-3' & 5'-ATCTTTTGGGGTCCGTCAACT-3' \\
TCF & 5'-TGACCCATCTCAGAAGCAG-3' & 5'-GCAGAGAGGAGGTTGACTTTC-3' \\
MMP & 5'-TGCCCACCGTCCTTTCTTGTT-3' & 5'-GCTGACTTGACTCATGGCT-3' \\
CT1 & 5'-CACAAAGCAGCCTTGCAGAA-3' \\
FBC & 5'-AACCCGAGGTATGCTTATCT-3' & 5'-AGAGCAGGCAGCATAGCAGTG-3' \\
$\beta$-actin & 5'-GTGGGCGCCCAGGCACCA-3' & 5'-CCAGTTCTTCATTGCATTGC-3' \\
\hline
\end{tabular}

SRPK-1, serine-arginine protein kinase-1; TCF, T-cell factor; MMP, matrix metalloproteinase; CT1, collagen type I; FBC, fibronectin.

purchased from Shanghai Slack Experimental Animals Co., Ltd. (Shanghai, China). All mice were housed in a temperature-controlled facility at $23 \pm 1^{\circ} \mathrm{C}$ and treated in accordance with the Guide for the Care and Use of Laboratory Animals of Tianjin Chest Hospital (Tianjin, China). Mice were maintained at a 12-h light/dark cycle with free access to food and water. The mice were implanted with NSCLCDVECs and were divided into two groups, which received ChanSRPK-1 or PBS treatment ( $\mathrm{n}=50$ in each group). Treatment was started 7 days post-tumor implantation, when the tumor diameter reached $6-8 \mathrm{~mm}$. The tumor-bearing mice were intravenously injected with ChanSRPK-1 (400 ng), with the same volume PBS injected in the control group mice. The treatment was continued for 14 days (one injection per day). The tumor volumes were calculated according to a previous study (21).

Statistical analysis. All data are presented as the means \pm standard error of the mean of triplicate experiments. Unpaired data was analyzed using Student's t-test and comparisons of data between multiple groups were analyzed by one-way analysis of variance (GraphPad version 5.0; GraphPad Software, Inc., La Jolla, CA, USA). The Kaplan-Meier test was used to estimate survival during the 120 -day observation period. $\mathrm{P}<0.05$ was considered to indicate a statistically significant difference.

\section{Results}

Construction of a chimeric antibody targeting SRPK-1 and determination of in vitro activity. The antibody targeting of SRPK1 was screened using a conventional approach and the constant region of antibody was replaced with a constant region from human antibody. To enhance the stability and half-life of the antibody targeting SRPK-1, the crystalline fragment was linked to the antibody targeting SRPK-1, and ChanSRPK-1 was analyzed. The structure of ChanSRPK-1, containing the crystalline fragment and cell-penetrating peptide to enhance stability and transmembrane ability, is shown in Fig. 1A. ChanSRPK-1 was expressed by pET-27b in Escherichia coli. As shown in Fig. 2B, the molecular weight of ChanSRPK-1 was $67.5 \mathrm{kDa}$, determined using SDS-PAGE gel electrophoresis. In addition, the affinity of ChanSRPK-1 for SRPK-1 was determined using ELISA and western blot analysis. The results (Fig. 1C) demonstrated that ChanSRPK-1 maintained a high affinity with SRPK-1 in the ELISA assay. A band at $\sim 67.5 \mathrm{kDa}$ was confirmed by SDS-PAGE gel electrophoresis, and the specific binding to SRPK-1 was confirmed using western blot analysis (Fig. 1D). These results indicated that ChanSRPK-1 was expressed at a high level and had the capacity to bind with SRPK-1.

In vitro effects of ChanSRPK-1 on the expression of SRPK-1 in NSCLC cells. In order to confirm the in vitro effects of ChanSRPK-1 (400 mg/ml) on the expression of SRPK-1 in NSCLC cells, NSCLCDVECs and MRC-5 cells were analyzed using RT-qPCR analysis and ELISA. The results (Fig. 2A and B) showed that the expression of SRPK-1 was upregulated in the NSCLCDVECs, compared with that in the MRC-5 human lung cells. In addition, the data (Fig. 2C) showed that ChanSRPK-1 treatment neutralized the expression of SRPK-1 in a dose-dependent manner (200-2,000 ng/ml). ChanSRPK-1 treatment also neutralized the expression of SRPK-1 in a time-dependent manner (24, 48, 72 and $96 \mathrm{~h}$; Fig. 2 C). The in vitro effect of ChanSRPK-1 on NSCLCDVEC growth was also examined. The results (Fig. 2D) showed that ChanSRPK-1 (400 mg/ml) suppressed NSCLCDVEC growth from $48 \mathrm{~h}$. These data suggested that the expression of SRPK-1 was increased in NSCLCDVECs, and that ChanSRPK-1 decreased the expression of SRPK-1 in NSCLCDVECs and inhibited growth.

In vitro inhibitory effects of ChanSRPK-1 on migration and invasion in NSCLC. The expression of SRPK-1 has been associated with the prognosis of human cancer, as TCF has been shown to promote the migration and invasion of NSCLC cells (15). Although the role of SRPK-1 in NSCLC cells has been investigated, the role of ChanSRPK-1 in the inhibition of migration and invasion in NSCLC cells has not been reported previously (21). To investigate the inhibitory effects of ChanSRPK-1 on NSCLCDVECs, cell viability, migration and invasion were analyzed. The results (Fig. 3A) showed that the viability of NSCLCDVECs was significantly decreased in the ChanSRPK-1-treated groups (200, 400 and $1,000 \mathrm{mg} / \mathrm{ml}$ for $48 \mathrm{~h}$ ), compared with that of the MRC-5 cells. In addition, the results (Fig. $3 \mathrm{~B}$ and $\mathrm{C}$ ) indicated that the presence of SRPK-1 markedly promoted NSCLCDVEC migration and invasion at doses of 200,400 and 1,000 mg/ml for $24 \mathrm{~h}$, compared with the untreated group. By contrast, it was demonstrated that $200-1,000 \mathrm{ng} / \mathrm{ml}$ of ChanSRPK-1 markedly inhibited the migration, invasion and apoptosis of NSCLCDVECs (Fig. 3D-F). Of note, $400 \mathrm{mg} / \mathrm{ml}$ of 
A

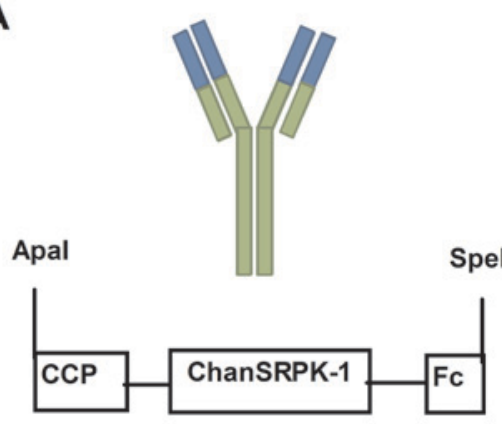

C

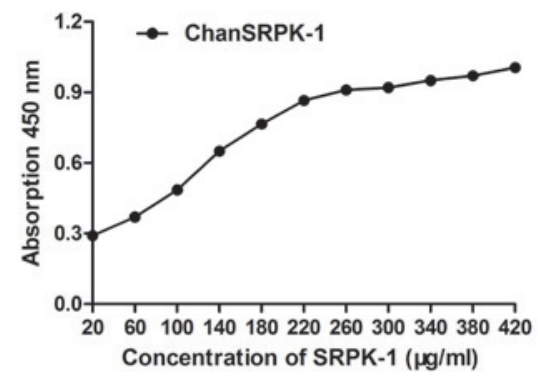

B

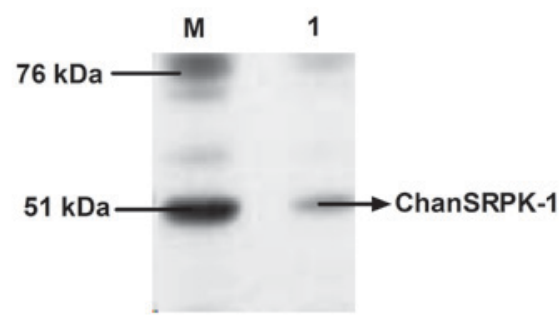

D

\section{Control SRPK-1}

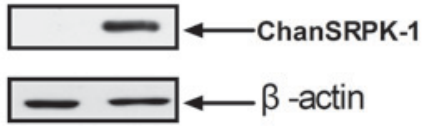

Figure 1. Characteristics of ChanSRPK-1. (A) Structure of the chimeric antibody of ChanSRPK-1. ChanSRPK-1 was aggrandized with CCP and Fc by genetic engineering. (B) SDS-PAGE analysis of purified ChanSRPK-1 under reducing conditions. Lane 1, molecular weight protein markers; lane 2, ChanSRPK-1. (C) Specific interaction of ChanSRPK-1 with SRPK-1. (D) Specificity of ChanSRPK-1 was analyzed using western blot analysis. SEPK-1, serine-arginine protein kinase-1; ChanSRPK-1, chimeric antibody target for SRPK-1; Fc, crystalline fragment; CCP, cell-penetrating peptide.

A

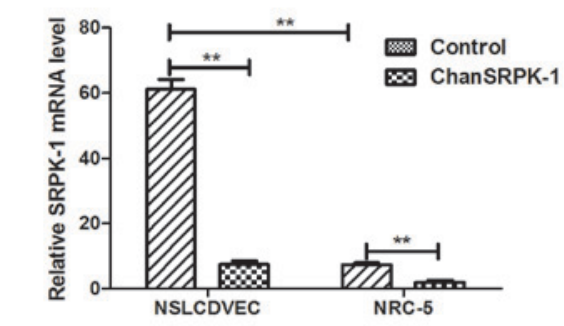

C

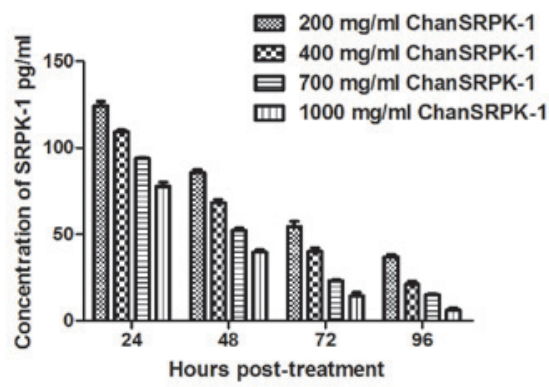

B

D
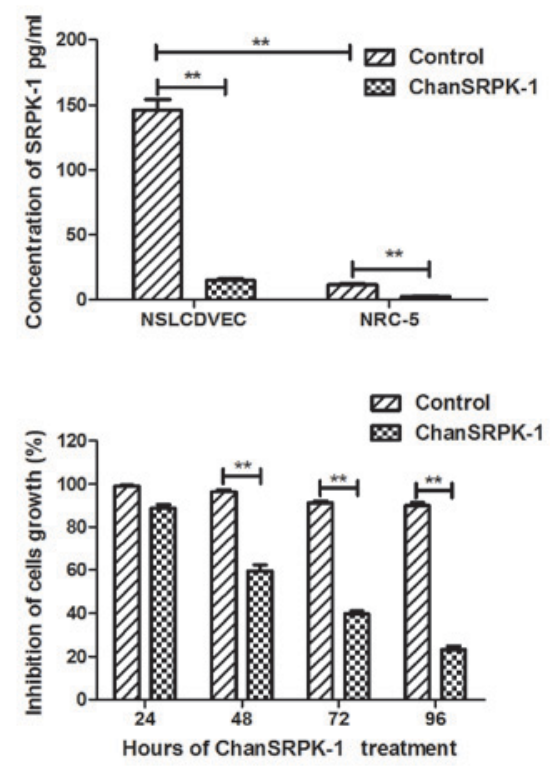

Figure 2. Effects of ChanSRPK-1 on the expression of SRPK-1 and growth of NSCLCDVECs. (A) Analysis of mRNA expression of SRPK-1 in NSCLCDVECs and MRC-5 cells pre- and post-treatment with ChanSRPK-1. (B) Detection of SRPK-1 levels in NSCLCDVECs and MRC-5 cells pre- and post-treatment with ChanSRPK-1. (C) Effects of time and dose in treatment with ChanSRPK-1 on levels of SRPK-1 in NSCLCDVECs. (D) Inhibitory effects of ChanSRPK-1 on NSCLCDVECs at a dose of $400 \mathrm{mg} / \mathrm{ml}$. ${ }^{*} \mathrm{P}<0.05$ and ${ }^{* *} \mathrm{P}<0.01$ between drug-treated and control group. SEPK-1, serine-arginine protein kinase-1; ChanSRPK-1, chimeric antibody target for SRPK-1; NSCLCDVES, non-small cell lung cancer-derived vascular endothelial cells.

ChanSRPK-1 was sufficient to inhibit the migration and invasion of NSCLCDVECs. These results suggested that ChanSRPK-1 not only affected cell viability, but also inhibited the migration and invasion of NSCLCDVECs.
Effects of ChanSRPK-1 on NSCLCDVECs by targeting TCF and in vivo efficacy in NSCLC-bearing mice. To determine the efficacy of ChanSRPK-1 for NSCLC treatment, the present study analyzed the expression of TCF, MMP, CT1 

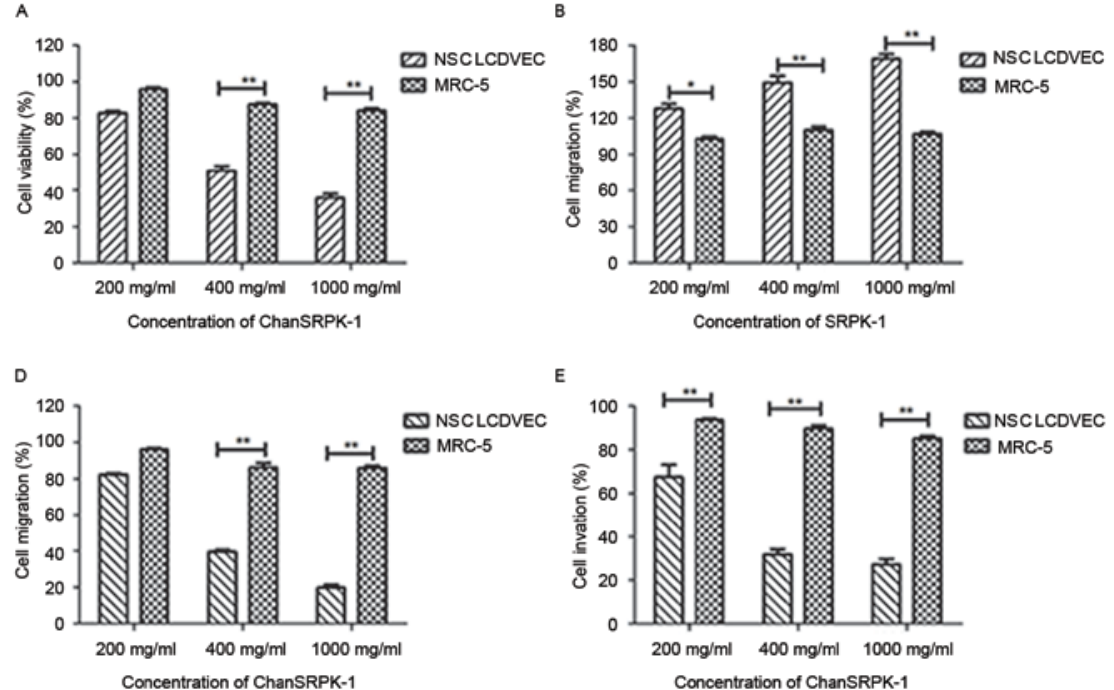

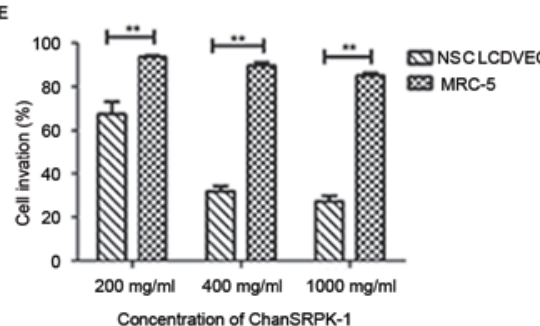

c
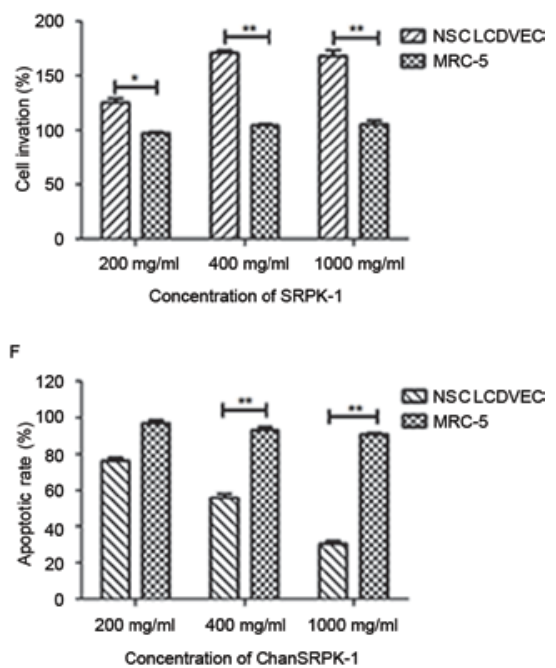

Figure 3. Effects of ChanSRPK-1 on the viability, migration, invasion and apoptosis of NSCLCDVECs. (A) Cell viabilities were significantly altered in ChanSRPK-1-treated NCSLCDVECs at different doses, compared with MRC-5 cells. (B) SRPK-1 promoted the migration of NSCLCDVECs following exposed to $200 \mathrm{mg} / \mathrm{ml}$, compared with control cells. (C) SRPK-1 promoted the invasion of NSCLCDVECs exposed to $200 \mathrm{mg} / \mathrm{ml}$, compared with control cells. (D) ChanSRPK-1 inhibited migration of NSCLCDVEC after exposure $200 \mathrm{mg} / \mathrm{ml}$, compared with control cells. (E) ChanSRPK-1 inhibited the invasion of NSCLCDVECs following exposure to $200 \mathrm{mg} / \mathrm{ml}$, compared with control cells. (F) ChanSRPK-1 induced the apoptosis of NSCLCDVECs following exposure to $400 \mathrm{mg} / \mathrm{ml}$, compared with control cells. ${ }^{*} \mathrm{P}<0.05$ and ${ }^{* *} \mathrm{P}<0.01$. SEPK-1, serine-arginine protein kinase-1; ChanSRPK-1, chimeric antibody target for SRPK-1; NSCLCDVES, non-small cell lung cancer-derived vascular endothelial cells.

A

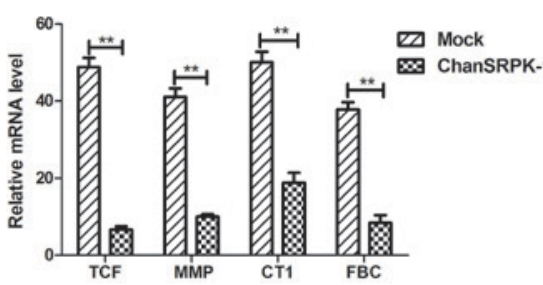

D

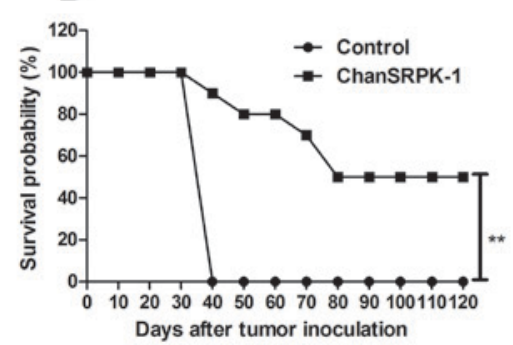

B
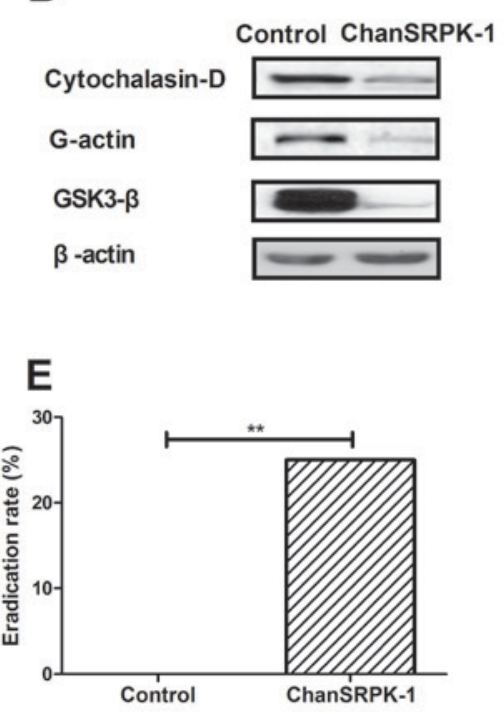

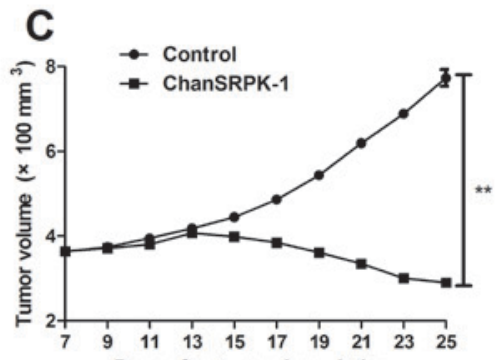

Days after tumor inoculation

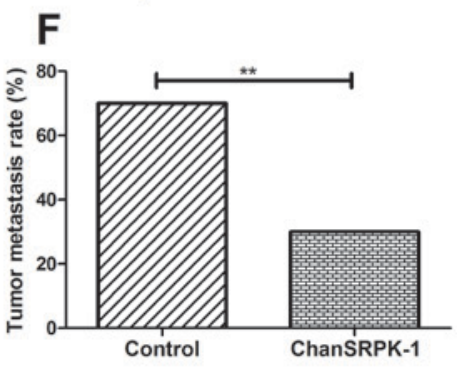

Figure 4. Beneficial therapeutic effects and inhibitory migration effects of ChanSRPK-1 in NSCLC-bearing mice. (A) ChanSRPK-1 had a beneficial effect on protein expression of tumor metastasis-associated TCF, MMP, CT1 and FBC in tumor-bearing mice, compared with control treatment. (B) ChanSRPK-1 decreased expression levels of Cytochalasin-D and G-actin, and the phosphorylation of GSK3- $\beta$ in tumors, compared with the untreated group. (C) ChanSRPK-1 treatment significantly inhibited tumor cell growth in NSCLC-bearing mice. (D) Survival was prolonged following treatment with ChanSRPK-1 over a 120-day observation period, compared with survival in the control group. (E) Eradication rate of NSCLC was enhanced following treatment with ChanSRPK-1. (F) Metastatic rate of NSCLC was decreased following treatment with ChanSRPK-1. The Kaplan-Meier test was used to estimate survival during the 120-day treatment period. ${ }^{*} \mathrm{P}<0.05$ and ${ }^{* *} \mathrm{P}<0.01$. SEPK-1, serine-arginine protein kinase-1; ChanSRPK-1, chimeric antibody target for SRPK-1; NSCLCDVES, non-small cell lung cancer-derived vascular endothelial cells; TCF, $\beta$-catenin/T-cell factor; MMP, matrix metalloproteinase; CT1, collagen type I; FBC, fibronectin; GSK3- $\beta$, glycogen synthase kinase 3- $\beta$.

and FBC. As shown in Fig. 4A, the mRNA expression levels of TCF, MMP, CT1 and FBC in the NSCLCDVECs were significantly higher, compared with those in the ChanSRPK-1-treated cells. The difference in the expression of migration-promoting proteins between the ChanSRPK-1 and control groups was also determined to be statistically significant using a paired t-test $(\mathrm{P}<0.01)$. A previous study showed that upregulation in the phosphorylation level of 
GSK3- $\beta$ by SRPK-1 was correlated with tumor cell migration (15). In the present study (Fig. 4B), it was found that the downregulation in the expression of Cytochalasin-D and G-actin, and the phosphorylation of GSK3- $\beta$ were significantly different in the ChanSRPK-1-treated tumor cells, compared with those in the control. In addition, NSCLC-bearing mice were used to determine the in vivo antitumor efficacy of ChanSRPK-1. As shown in Fig. 4C, tumor size was significantly suppressed in xenograph mice in the ChanSRPK-1-treated group, compared with those in the control group. In addition, long-term (120 day) survival was preceded following treatment with ChanSRPK-1. The data (Fig. 4D) revealed that treatment with ChanSRPK-1 $(n=10$ in each group) prolonged the survival of NSCLC-bearing mice, compared with control mice. Furthermore, it was demonstrated (Fig. 4E) that treatment with ChanSRPK-1 significantly protected tumor-bearing mice via eradicating tumors. As demonstrated in Fig. 4F, it was found that ChanSRPK-1 inhibited NSCLC tumor metastasis compared with PBS-treated mice. The data showed that the inhibitory effect of ChanSRPK-1 on tumor metastasis in NSCLC-bearing mice was enhanced, compared with that in the control. These results suggested that ChanSRPK-1 suppressed the migration-promoting protein and contributed to beneficial efficacy in NSCLC-bearing mice.

\section{Discussion}

Lung cancer is a respiratory disease leading to the majority of cancer-associated mortality in the world owing to air contamination (22). At present, NSCLC accounts for $\sim 80 \%$ of lung cancer cases and its incidence is increasing. It includes adenocarcinoma, which comprises large cell carcinoma and squamous cell carcinoma (5-7). The high mortality rates of NSCLC present a challenge, and a rapidly increasing trend has been observed, which has gradually become a focus of public opinion and a significant burden on human health (23). The difficulty in the early detection of NSCLC is the primary reason for lower survival rates in advanced NSCLC (24). The majority of patients with NSCLC are at an advanced stage of lung cancer at diagnosis (23). In addition, conventional therapeutic techniques show poor efficacy for NSCLC, and recurrence and metastasis are frequently observed clinically. Statistical data have also shown that the survival rates of patients with NSCLC, in terms of the overall 5-year survival rate, are poor (25). Therefore, the identification of more efficient anti-NSCLC agents is required for patients with NSCLC in preclinical and clinical trials.

Growth, migration and invasion in NSCLC are the most important drivers for tumor metastasis and poor survival rates in treatment and recurrence for patients with $\operatorname{NSCLC}(10,11)$. Previous studies have reported that SRPK-1-encoding transcripts are ubiquitously expressed in several human tissues, and higher expression levels of SRPK-1 in the precise cellular localization of NSCLC, pancreatic carcinoma, colon cancer and breast cancer have been reported previously $(13,15)$. It has been identified that the majority of SRPK-1 protein is expressed exclusively within NSCLCDVECs and that SRPK-1 is positively correlated with the grade of cancer progression. Furthermore, the downregulation of SRPK-1 increases the apoptosis of tumor cells (17). These results suggest that therapeutic agents, through suppressing the activity of SRPK-1, may be effective as a potential single anticancer agent or an adjuvant agent in combination with conventional therapeutic regimens.

In the present study, the design involved construction of a Chan-SRPK-1 for NSCLC therapy via inhibition of growth, migration and invasion. The expression of SRPK-1 and inhibitory effects on NSCLCDVECs were also examined in vitro and in vivo. Lehman et al (12) reported that two major kinases responsible for the protein phosphorylation of mitogen-activated protein kinase (MAPK)3 and MAPK1 were reduced through inhibited phosphorylation levels of GSK3- $\beta$. In the present study, the mRNA expression levels of the TCF, MMP, CT1 and FBC tumor cell metastasis-associated proteins were significantly decreased in NSCLCDVECs treated with ChanSRPK-1, compared with levels in untreated cells.

The ability of SRPK-1 to enhance proliferation and survival via the regulation of multiple signaling pathways in tumor cells has been exploited and suggested as an opportunity to develop novel anticancer therapeutics targeting SRPK-1 (26). A previous study reported that SRPK-1 regulates migration and growth in various cancer cells (27). In the present study, it was shown that the expression of SRPK-1 was superfluous in NSCLCDVECs, compared with normal MRC-5 lung cells. It was found that ChanSRPK-1 interrupted the signaling pathway involved in tumor cell growth, migration and invasion regulated by SRPK-1. In addition, ChanSRPK-1 treatment at a concentration of $400 \mathrm{ng} / \mathrm{ml}$ for $24 \mathrm{~h}$ markedly suppressed NSCLCDVEC migration, compared with that in the untreated cells in vitro, and inhibited tumor metastasis in tumor-bearing mice in vivo. Primary data analysis of tumor diameter and long-term survival showed that injection with ChanSRPK-1 once a day led to tumor regression and resulted in a survival rate of $50 \%$ in a 120 -day period in tumor-bearing mice. Data also indicated that treatment with ChanSRPK-1 against NSCLC was sufficient to partially protect the animals via the eradication of tumors in experimental mice, which translated into long-term survival and tumor-free living in the mice of the lung cancer model.

In conclusion, the present study introduced ChanSRPK-1 as an anticancer candidate via once daily administration through intravenous injection. In the NSCLC mouse model, the results demonstrated that, ChanSRPK-1 downregulated the expression of SRPK-1, which led to the reversal of TCF-induced cell migration. Taken together, the data obtained in the present study suggested the beneficial effects of cellular targeted therapy, which can assist in further elucidating the clinical value of applying such a regimen.

\section{References}

1. Blair HA and Deeks ED: Albumin-bound paclitaxel: A review in non-small cell lung cancer. Drugs 75: 2017-2024, 2015.

2. DeCotiis C, Hu Y, Greenberg AK, Huie M, Tsay JC, Pass H, Goldberg JD and Rom WN: Inflammatory cytokines and non-small cell lung cancer in a CT-scan screening cohort: Background review of the literature. Cancer Biomark 16: 219-233, 2016.

3. Keating GM: Nivolumab: A review in advanced squamous non-small cell lung cancer. Drugs 75: 1925-1934, 2015. 
4. van der Wekken AJ, Saber A, Hiltermann TJ, Kok K, van den Berg A and Groen HJ: Resistance mechanisms after tyrosine kinase inhibitors afatinib and crizotinib in non-small cell lung cancer, a review of the literature. Crit Rev Oncol Hematol 100: 107-116, 2016.

5. Brody H: Lung cancer. Nature 513 (Suppl): S1, 2014.

6. Moro-SibilotD,SmitE, de Castro Carpeño J,Lesniewski-KmakK, Aerts JG, Villatoro R, Kraaij K, Nacerddine K, Dyachkova Y, Smith KT, et al: Non-small cell lung cancer patients with brain metastases treated with first-line platinum-doublet chemotherapy: Analysis from the European FRAME study. Lung Cancer 90: 427-432, 2015.

7. Barnett SA, Downey RJ, Zheng J, Plourde G, Shen R, Chaft J, Akhurst T, Park BJ and Rusch VW: Utility of routine PET imaging to predict response and survival after induction therapy for non-small cell lung cancer. Ann Thorac Sur 101: 1052-1059, 2016.

8. Xie FJ, Lu HY, Zheng QQ, Qin J, Gao Y, Zhang YP, Hu X and Mao WM: The clinical pathological characteristics and prognosis of FGFR1 gene amplification in non-small-cell lung cancer: A meta-analysis. OncoTargets Ther 9: 171-181, 2016.

9. Lim SH, Sun JM, Lee SH, Ahn JS, Park K and Ahn MJ: Pembrolizumab for the treatment of non-small cell lung cancer. Expert Opin Biol Ther 16: 397-406, 2016

10. MüllerB,BovetM,Yin Y,StichelD,MalzM,González-Vallinas M, Middleton A, Ehemann V, Schmitt J, Muley T, et al: Concomitant expression of far upstream element (FUSE) binding protein (FBP) interacting repressor (FIR) and its splice variants induce migration and invasion of non-small cell lung cancer (NSCLC) cells. J Pathol 237: 390-401, 2015.

11. Zhao Q, Yue J, Zhang C, Gu X, Chen $\mathrm{H}$ and $\mathrm{Xu}$ L: Inactivation of M2 AChR/NF- $\mathrm{BB}$ signaling axis reverses epithelial-mesenchymal transition (EMT) and suppresses migration and invasion in non-small cell lung cancer (NSCLC). Oncotarget 6 : 29335-29346, 2015.

12. Lehman M: Improving therapeutic outcomes in non-small cell lung cancer not suitable for curative intent therapy- A review of the role of radiation therapy in an era of increasing systemic therapy options. Clin Oncol (R Coll Radiol) 28: 327-333, 2016.

13. Polo V, Zago G, Frega S, Canova F, Bonanno L, Favaretto A Bonaldi L, Bertorelle R, Conte P and Pasello G: Non-small cell lung cancer in a very young woman: A case report and critical review of the literature. Am J Case Rep 16: 782-789, 2015.

14. Li XH, Song JW, Liu JL, Wu S, Wang LS, Gong LY and Lin X: Serine-arginine protein kinase 1 is associated with breast cancer progression and poor patient survival. Med Oncol 31: 83, 2014.

15. Liu H, Hu X, Zhu Y, Jiang G and Chen S: Up-regulation of SRPK1 in non-small cell lung cancer promotes the growth and migration of cancer cells. Tumour Biol 37: 7287-7293, 2016.
16. Krishnakumar S, Mohan A, Kandalam M, Ramkumar HL, Venkatesan N and Das RR: SRPK1: A cisplatin sensitive protein expressed in retinoblastoma. Pediatr Blood Cancer 50: 402-406, 2008.

17. Mavrou A, Brakspear K, Hamdollah-Zadeh M, Damodaran G, Babaei-Jadidi R, Oxley J, Gillatt DA, Ladomery MR, Harper SJ, Bates DO and Oltean S: Serine-arginine protein kinase 1 (SRPK1) inhibition as a potential novel targeted therapeutic strategy in prostate cancer. Oncogene 34: 4311-4319, 2015.

18. Hayes GM, Carrigan PE and Miller LJ: Serine-arginine protein kinase 1 overexpression is associated with tumorigenic imbalance in mitogen-activated protein kinase pathways in breast, colonic, and pancreatic carcinomas. Cancer Res 67: 2072-2080, 2007.

19. Nishio M, Horai T, Horiike A, Nokihara H, Yamamoto $\mathrm{N}$, Takahashi T, Murakami H, Yamamoto N, Koizumi F, Nishio K, et al: Phase 1 study of lenvatinib combined with carboplatin and paclitaxel in patients with non-small-cell lung cancer. Br J Cancer 109: 538-544, 2013.

20. Sultani M, Mokhtari Azad T, Eshragian M, Shadab A, Naseri M, Eilami O and Yavarian J: Multiplex SYBR green real-time PCR assay for detection of respiratory viruses. Jundishapur J Microbiol 8: e19041, 2015.

21. Han S, Bui NT, Ho MT, Kim YM, Cho M and Shin DB: Dexamethasone inhibits TGF- $\beta 1$-induced cell migration by regulating the ERK and AKT pathways in human colon cancer cells via CYR61. Cancer Res Treat 48: 1141-1153, 2016.

22. Fenton-Ambrose L and Kazerooni EA: Preventative care: Lung-cancer screens now worth the cost. Nature 514: 35, 2014.

23. Kim DS, Park KM, Won YS, Kim JY, Lee JK, Kim JG, Oh ST, Jung SS and Kang WK: Occurrence and prognosis of symptomatic venous thromboembolism in colorectal cancer surgery patients. Vasc Specialist Int 30: 49-55, 2014.

24. Tauhardt E, Reissig A, Winkens T and Freesmeyer M: Early detection of disease progression after palliative chemotherapy in NSCLC patients by (18)F-FDG-PET. Nuklearmedizin 53: 197-204, 2014.

25. Gold M, Dunn LB, Phoenix B, Paul SM, Hamolsky D, Levine JD and Miaskowski C: Co-occurrence of anxiety and depressive symptoms following breast cancer surgery and its impact on quality of life. Eur J Oncol Nurs 20: 97-105, 2016.

26. Huang W, Chang HY, Fei T, Wu H and Chen YG: GSK3 beta mediates suppression of cyclin D2 expression by tumor suppressor PTEN. Oncogene 26: 2471-2482, 2007.

27. Yoon C, Kim D, Kim S, Park GB, Hur DY, Yang JW, Park SG and Kim YS: MiR-9 regulates the post-transcriptional level of VEGF165a by targeting SRPK-1 in ARPE-19 cells. Graefes Arch Clin Exp Ophthalmol 252: 1369-1376, 2014. 